Bruce W. Kennedy, MS, RLATG, CMAR, CPIA, Column Coordinator

\title{
The value of quizzes
}

In recent Fruits of Education columns, I have shared the curriculum of my collegelevel lab animal technology class for veterinary technicians and discussed ways to assess knowledge and skills. I regularly use quizzes to help determine what they are learning and absorbing. Quizzes have several advantages compared to other types of assessments: quizzes are quicker to execute, quicker to grade, quicker to return with feedback and less stressful than major examinations.

In a classroom scenario, such as an academic environment or a long-term course for certification or professional examination, quizzes can be used to promote learning and retention of knowledge throughout the course. This is in contrast to the 'big' exam, with all of its associated consternation, which represents the sum of a student's learning and preparation.

Remember our days in secondary school and college when we had a mid-term or two before the final examination in a course? Truth be told, we procrastinated week after week, putting off continuous studying that should have occurred. We prepared for the test by cramming the material into our short-term memory on the night (perhaps throughout the night) before the test. As an alternative, since I care about the welfare of my students as well as lab animals, I present weekly quizzes throughout a course. These usually last 10-15 minutes and are based on the material of the previous week. The questions are true-or-false, multiple choice, short-answer essay prompts and similar measures that portray what happens in daily vivarium life. When working with animals, rarely are we presented with questions that have simple, single answers; typically the response is a hybrid of answers. And it's been shown that this approach of

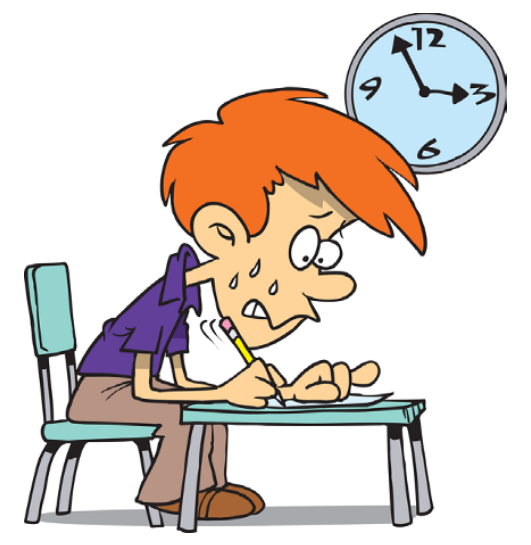

mixing the questions is an effective way to both teach material and assess knowledge.

I take my quizzing one distinct step further: my students and I immediately grade their quizzes in class. I don't take them away and return them at the next class meeting; rather, students keep their own quizzes and we go through each question, grading, answering and discussing it in class. We confirm right answers and analyze wrong answers to understand why they are wrong. Sometimes a student provides a rationale explaining why a wrong answer might be correct under certain circumstances. For that I give credit because the quiz is about the learning and not the grade. The ensuing discussions demonstrate critical thinking on the part of that student, and all students listening can benefit as well.

There are additional advantages to using quizzes to teach and assess knowledge. Having several small quizzes instead of one large exam can dilute the pressure and negative impact of a poor performance if a student has a bad day. Students know to expect a quiz assessment at each class meeting, so they study and prepare over time rather than all at once. Taking frequent, lowimpact quizzes can diminish test-taking anxieties and prepare students for major tests such as those required by the American Association for Laboratory Animal Science for technicians and managers, the American College of Laboratory Animal Medicine for veterinarians, the Academy of Surgical Research for surgical technicians and Public Responsibility in Medicine and Research for IACUC administrators. Offering quizzes before and after a training session, with material presented in between, gives participants immediate feedback on the effectiveness of a training session and the knowledge they have gained from it. And if participants opt out of a presentation, for whatever reason, they can take the quiz to confirm that they do indeed know the teaching objectives that were designed for that session.

So far, my description and usage of quizzes have related to factual knowledge; however, quizzing can also build and assess a student's competency with a process or technique. Breaking up learning and assessment into chunks, with quizzes, can provide the scaffolding or ladder rungs for a student to demonstrate competency of a major, multiple-part procedure.

Current research shows that the brain is a quirky learning machine, demanding units of learning that are spaced apart. Learning snippets and taking quizzes might better match the needs of our brain for assimilating information. Studies show that this helps both athletes and students improve their skills and test scores, respectively. Smaller mental packets of information, presented here and there, with time in between to process, seem to aid learning more effectively than does a massive burst of information. And, based on feedback from my own students, they agree. Thus, it is recommended that teachers and trainers incorporate quizzes as means to both teach and assess. 